\title{
Analysis on the Influence of Big Data Age on Consumers' Purchase Behavior and Marketing Innovation
}

\author{
Li Yao \\ School of Economics and Management, Tianjin University of Technology and Education \\ NO.1306, dagu south Road, Hexi District, Tianjin city \\ 15522545620@163.com
}

Key words: Big data, Consumer behavior, Marketing innovation.

\begin{abstract}
Big data refers to a collection of data that can not be captured, managed, and processed with conventional software tools within an affordable timeframe. Dissemination and application of big data has led to a series of changes of consumer behavior, which also put forward new requirements for the enterprise's marketing model enterprises. Enterprises should take advantage of the big data, tap the value of data, change marketing concepts and improve business efficiency.
\end{abstract}

\section{大数据时代对消费者购买行为及营销创新的影响分析}

\author{
李瑶 \\ 天津职业技术师范大学经济与管理学院＼cjkstart河西 天津 中国 \\ 天津河西区大沽南路1306号
}

15522545620@163.com

关键字: 大数据; 消费者行为; 营销创新

摘要.大数据是指无法在可承受的时间范围内用常规软件工具进行捕捉、管理和处理的数据集 合。大数据的广泛传播和应用导致消费者行为发生了一系列变化, 这对企业的营销模式也提 出了新要求, 企业须利用大数据的优势, 挖掘数据价值, 转变营销观念, 提高企业运营效率。

\section{1. 引言}

麦肯锡环球研究院于 2011年5月发布了《大数据：创新、竞争和生产力的下一个前沿》报 告。该报告中指出“大数据可以发挥重要的经济作用, 不但有利于私人商业活动, 也有利于国 民经济和民生。数据可以为世界经济创造重要价值, 提高企业和公共部门的生产率和竞争力, 并为消费者创造大量的经济剩余。”今年伊始, 贵州省政府印发了《贵州省数字经济发展规划 (2017-2020年)》，成为全国首个发布的省级数字经济发展专项规划。2017年3月5日，李克强 总理两会所作的政府工作报告中强调“深入推进“互联网+”行动和国家大数据战”, “加快大数 据、云计算、物联网应用, 以新技术新业态新模式, 推动传统产业生产、管理和营销模式变 革”。这是大数据自2014年成为国家战略以来连续第四年被写入政府工作报告。

\section{1 大数据的内涵及应用}

根据维基百科的定义, 大数据是指无法在可承受的时间范围内用常规软件工具进行捕捉、 管理和处理的数据集合。在维克托.迈尔-舍恩伯格及肯尼斯. 库克耶编写的《大数据时代》中 大数据指不用随机分析法 (抽样调查) 这样的捷径, 而采用所有数据进行分析处理。目前大 
数据具有以下 4 个特征 $(4 \mathrm{~V})$ ：一是数据的量大（Volume）, 到 2012 年, 数据量 $\mathrm{TB}$ 级别上升 到 $\mathrm{PB} 、 \mathrm{~EB}$ 甚至是 $\mathrm{ZB}$; 二是数据种类繁多（Variety），比如音频、视频、图片、日志等，大 体上分为结构化和半结构化两种类型。三是信息的处理速度快（Velocity）, 因为数据数量庞 大且高速实时流转, 这对数据处理的速度提出了更高的要求。三是价值密度低(Value)。价值 密度的高低与数据总量的大小成反比。因为数据量的庞大, 数据中有价值的信息量显得较小, 所以如何在大量数据中提取有价值的信息是数据分析技术上的新挑战。大数据在城市管理, 农林工商，居民生活上都有广泛应用。

\section{2 消费者购买行为分析}

消费者购买行为的研究核心是消费者如何做出购买决策。消费者购买决策是指消费者谨 慎地评价某一产品、品牌或服务的属性并进行选择、购买能满足某一特定需要的产品的过程。 消费者做出购买决策的过程包括5步：需求确认、购买前搜索、替代品评估、决定购买、购后 评价。

需求确认。在现实生活中，当消费者面对某个问题时，“需求”才可能出现。从消费者角 度来看, 有两种需求方式: 一些消费者是真实状态方式, 这些消费者现有的产品或服务未能 满足他们需求。比如手机有噪音, 影响正常通话消费者就会就会考虑换一部手机。相反的, 其他消费者就是需求状态方式, 对这种消费者来说一个他们感兴趣的新产品就能刺激起他们 的需求。

购买前搜索。当消费者觉察到能够通过购买或消费某种产品或服务时，消费者就会开始 购买前的信息搜索。在搜索过程中, 最先发挥影响作用的是消费者之前的经验, 消费者过去 的经验被当做信息的内部来源。大部分情况下, 消费者都是同时依靠内部信息来源和外部信 息来源即商业信息做出购买决策。

替代品评估。消费者在搜寻信息过程中可能得到的某些信息是相矛盾的或重复的，因此 还要进行分析、评估和选择, 这是决策过程中的决定性环节。消费者在评价潜在的可替代品 时, 倾向于根据自己做出的品牌清单做选择; 另一方面每个消费者对产品各种性能的重视程 度不同，所以会根据自己最重视的产品性能做出决定。

购买行为。购买行为指消费者购买商品时的行为，消费者的购买行为一般分为三类：试 探性购买、重复性购买和长期忠实性购买。在购买过程中, 产品的质量, 商家的服务态度及 物流的速度等都会影响到消费者的购买行为。

购后评价。消费者对商品或服务的评价实际上就是消费者将购得的商品性能与自身的期 望之间的差距。当实际情况与消费者预期相匹配, 消费者则持中立态度; 实际情况超出预期, 导致积极性预期不匹配即消费者满意; 实际低于预期时, 导致消费者消极性不匹配以及消费 者不满意。

\section{2. 大数据对消费者购买行为的影响}

随着大数据时代的到来，人们获得信息的渠道越来越广泛、方式越来越便捷，对产品的 考量更加全面, 为消费者购买决策的制定提供了更加充足的理由。消费者在决策时也逐渐趋 于理性。

\section{1 消费者消费行为更加理性}

在传统商业中，人们获取商品信息主要依靠报纸、电话、人们的口碑这些传统传播媒介。 在如今的大数据网络时代中, 人们可以通过微博、微信、贴吧等发表自己对产品使用的看法。 各种各样的媒介广泛传播, 数据无处不在, 消费者可以快捷方便随时随地了解到产品数据, 从而做出合理的购买决策, 提高购买效率同时降低购买成本。这些评价影响着其他消费者对 该产品的初步印象, 所以这种评价模式直接影响了潜在的购买者是否决定购买该产品。这种 
影响传播越广泛，影响消费者的数量越大，对产品销售情况影响越大，严重时可能会决定一 个产品的存亡。

\section{2 消费者对品牌的依赖度降低}

在工业化时代，品牌往往代表着质量，包含着人们的崇拜错误!未找到引用源。。但在大 数据时代, 消费者可以轻松的了解到关于商品全方位的信息, 认识到商品的真正质量如何, 能否满足自己的需要。所以品牌便不再是衡量商品质量的唯一标准。而且很多大品牌也会将 产品外包来降低生产成本, 品牌产品加工制造的地方不同, 质量上也会有一定差异。同样的, 虽然各产品在品牌上有所差异, 但来自一个生产地, 质量上不会有太大差异。由于数据的广 泛和多样, 这种情况会被消费者得知, 消费者便不会过度迷信品牌, 而是更加理性的考虑自 己需要什么。

\section{3 消费者更加追求个性化和创新性}

在传统模式下，由于信息的闭塞，企业大规模生产，消费者即使有个性化的需求也很难 得到满足。在大数据时代, 信息变得通畅和便捷, 文娱活动的丰富, 人们的思维更加跳跃和 充满想象, 对于个性的追求越来越强烈。人们在购买产品时不但看重产品的质量, 个性化也 逐渐成为消费者决定购买的首要标准。因为产品已不单单是一件满足需求的产品, 产品的风 格和样式在一定层面上代表着购买者的个人形象，展示自己的个性错误!未找到引用源。。产 品的多样化也刺激着消费者对自由选择的需求。

\section{3. 大数据对营销创新的影响}

\section{1 精准营销}

在大数据时代，企业掌握了越来越多的用户数据，企业更加方便准确地分析出消费者的 偏好和习惯, 从而实施精准营销, 提升企业利润。这就要求企业首先要树立大数据的思维, 大数据思维是一种态度, 一种意识, 对大数据的重视就是对价值的重视错误!未找到引用源。。 企业必须重视信息的获取和更新, 建立多渠道获取信息, 逐渐将静态的数据转化为动态的数 据流。其次要求企业对消费者精准细分, 企业可以凭借先进的技术, 建立数据库, 采用数据 挖掘技术，通过对消费者的搜索、汶览和购买记录、社交媒体评论等的数据收集和整理，分 析消费者的心理和类型, 从而制定出一对一的个性营销计划错误!未找到引用源。。在这方面 做得比较好的如淘宝亚马逊, 通过对单个用户的数据分析, 精准推送商品信息, 刺激消费者 的潜在购买需求。

\section{2 网络营销}

大数据时代，传统媒介如报纸，杂志等对信息的传播作用明显降低，网络传播的广泛性 和便捷性已成为信息的主要传播形式，未来企业要着力利用好这一市场营销工具。网络的优 势就是传播信息和收集信息, 企业在开展营销时要充分利用全媒体的优势, 向消费者传递企 业的文化理念, 紧紧把握消费趋势。一方面企业可以通过大数据追踪消费者的行为习惯, 制 定有针对性的优惠和促销活动。另一方面企业通过网络收集销售数据，对销售数据的分析可 准确评估营销活动效果如何, 从而做出有针对性的方案修改, 提高销售量。总之, 大数据的 应用在网络营销中无处不在, 合理清晰的数据分析不但为企业做出管理决策提供了强有力的 支撑，而且提高了决策效率，降低了运营成本。

\section{3 以消费者为中心}

由于消费者的消费行为一方面更加理性，不再盲目崇拜品牌；另一方面也更加个性，对 商品的要求不仅是满足基本需求。对于产品信息也不像过去企业大力主动传播, 消费者被动 接受。在大数据时代, 消费者接受信息的渠道广泛，同时消费者自己也会主动搜寻，只要消 
费者根据自己的需要搜索必然会留下信息，企业生产本就是为了将产品通过交易到消费者手 中来获取利益。所以企业要以消费者为中心, 挖掘数据中的价值, 了解消费者对产品的需求, 对消费者进行定位, 帮助企业更好营销。因此, 以消费者为中心, 把握并利用好消费者的信 息将成为商业模式成功变革的保障。

\section{4. 总结}

一方面消费者的购买行为在大数据的影响下已经发生了巨大变化, 另一方面大数据也为 企业提供了营销创新的新视角和分析消费者行为的新方法。随着科技的不断进步, 大数据越 来越成为驱动企业创新的动力。但同时为避免大数据应用上的失误, 如隐私的界定, 在使用 大数据时还要提高个人的道德水平, 保证大数据在合理合法的范围内使用。

\section{致谢}

本文为天津职业技术师范大学科研发展基金资助项目（项目编号SK13-12）阶段性成果之

\section{References}

[1] Bai Shengjie. Big data age consumer behavior changes and the impact of business model changes [J]. Finance (Academic Edition), 2016, (23): 337.

[2] Zhang Chengsen, Yan Zhigao, Wang Shuangying, Zhu Hao.The analysis of Chinese consumer behavior based on the big data [J]. China Market, 2016, (27): $25+27$.

[3] Shi Lingjun. Changes in business model based on big data [J]. Jiangsu Science and Technology Information, 2015, (33): 28-30.

[4] Yin Shaoming. Consumer behavior of buying insight and marketing model changes based on big data [J]. China Business, 2015, (24): 8-11.

[5] Li Fu. Consumer behavior changes and the impact of business model changes based on big data[J]. China Circulation Economy, 2014, (10): 87-91.

[6] Li Wenlian, Xia Jianming. Business model innovation based on "big data" [J]. China Industrial Economy, 2013, (05): 83-95.

[7] Huang Shengmin, Liu Shan. Deconstruction and reconstruction of marketing system based on "Big Data" [J]. Modern Communication (Journal of Communication University of China), 2012, (11): 13-20. 